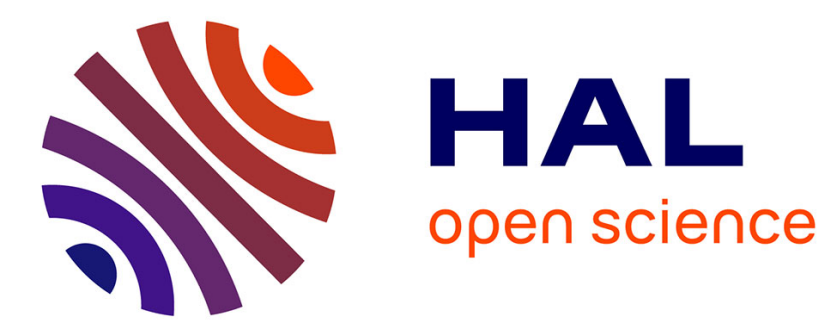

\title{
Retraites et développement territorial
}

Magali Talandier

\section{To cite this version:}

Magali Talandier. Retraites et développement territorial. Pour: enquêtes et témoignages, 2018, 1 (233), pp.80-87. 10.3917/pour.233.0080 . halshs-01896223

\section{HAL Id: halshs-01896223 https://shs.hal.science/halshs-01896223}

Submitted on 18 Dec 2018

HAL is a multi-disciplinary open access archive for the deposit and dissemination of scientific research documents, whether they are published or not. The documents may come from teaching and research institutions in France or abroad, or from public or private research centers.
L'archive ouverte pluridisciplinaire HAL, est destinée au dépôt et à la diffusion de documents scientifiques de niveau recherche, publiés ou non, émanant des établissements d'enseignement et de recherche français ou étrangers, des laboratoires publics ou privés. 


\section{Retraites et développement territorial \\ Article paru dans la revue POUR, numéro 233, sept. 2018}

Magali Talandier

Professeure en Aménagement et urbanisme, Université Grenoble Alpes, laboratoire PACTE

Magali.talandier@univ-grenoble-alpes.fr

L'augmentation du temps libre, l'allongement de la durée de vie, l'accélération des vitesses de déplacement, l'amélioration des niveaux de revenus des ménages... ont permis l'essor des mobilités durant plusieurs décennies et ont eu raison de la logique " un individu-un territoire ". C'est cette multi-appartenance territoriale, cette forme d'habiter poly-topique (Stock, 2006) qui explique, qu'aujourd'hui, la production et la consommation sont pour partie déterminées par des logiques géographiques différentes. Cette déconnexion spatiale et temporelle de l'offre et de la demande a fait émerger un fait nouveau et sans précédent pour l'économie des territoires : le " désajustement » entre la création de richesse et l'amélioration des conditions de vie des populations (Davezies, 2008). Ce désajustement entre croissance et développement à l'échelle infranationale s'explique par de puissants mécanismes de redistribution publique et privée entre les territoires. Ces flux de revenus irriguent les territoires indépendamment de leur capacité productive et constituent une ressource économique majeure et un levier d'action pour les territoires, parmi lesquels sont à compter les flux de retraites.

Dès lors, le développement économique local ne dépend plus exclusivement de la qualité de son système productif et dès les années 1990 des espaces éloignés de villes et peu impactés par la périurbanisation des ménages se repeuplent et enregistrent une croissance rapide des emplois et du revenu de leurs habitants. Ce "développement hors métropolisation" (Talandier, 2007) s'explique par l'essor d'une économie résidentielle et touristique, venue dans certains cas - alimentée voire supplantée une économie rurale agricole et/ou industrielle.

Les nouvelles mobilités temporaires ou résidentielles ont donc permis l'essor d'une nouvelle forme d'économie non exportatrice, appelée l'économie résidentielle.

\section{L'économie résidentielle, premier moteur économique des territoires}

Depuis une dizaine d'années, des travaux de recherche se développent autour de la notion d'économie résidentielle, reconnue en peu de temps comme l'un des moteurs majeurs du développement économique des territoires. Cette notion mobilise de plus en plus de chercheurs en France, en Europe, mais également dans quelques pays des Suds. 
Le terme d'économie résidentielle n'est pour autant toujours pas stabilisé et renvoie à des définitions en français, mais aussi en anglais (residential economy), qui peuvent varier d'un auteur à l'autre. L'économie résidentielle peut désigner un ensemble de flux de revenus que captent les territoires grâce à leurs aménités (acception considérée par exemple par Davezies, Talandier, Segesseman, Crevoisier). La notion est aussi utilisée pour qualifier les emplois créés grâce à la consommation locale des ménages (Aubert, Vollet, Dissart...). L'économie résidentielle, enfin, est parfois associée au marché de la construction résidentielle. Une autre notion, l'économie présentielle, est également apparue dans le paysage de l'aménagement au cours des dix dernières années (traduite en anglais par le terme in-place economy dans Talandier, 2012). Ce terme proposé par l'Insee suite aux travaux de C. Terrier sur la présence touristique regroupe les activités tournées vers la demande des ménages présents sur un territoire. Enfin, selon les auteurs, on peut voir apparaitre les termes de bases, sphères ou économie, les adjectifs " basique " ou " induite » associés à la notion de " résidentiel » et/ou " présentiel »... ce qui commence à faire un certain nombre de déclinaisons possibles !

Ici, nous considérons que l'économie résidentielle désigne l'une des bases économiques locales, à savoir l'un des moyens qui permettent aux territoires de de capter des richesses à l'extérieur. Ainsi, les bases économiques peuvent être regroupées en 4 grandes familles (Davezies, 2008) :

- la base résidentielle, comme nous l'avons évoquée, comprend les revenus captés par les territoires grâce à leurs atouts résidentiels. On comptabilise les pensions de retraites, les salaires des migrants-alternants qui résident dans le territoire mais $n$ 'y travaillent pas, les dépenses des touristes en hébergements marchands et non marchands ;

- la base productive privée ou base exportatrice comprenant les revenus issus d'activités qui se localisent pour produire et exporter. Ces revenus sont constitués des salaires et des bénéfices des non-salariés travaillant dans les secteurs exportateurs (industrie, agriculture, $R \& D$, une partie des services supérieurs aux entreprises...), des revenus des capitaux mobiliers et d'une partie des revenus fonciers et immobiliers ;

- la base publique comprend les traitements des fonctionnaires (fonction publique d'Etat, territoriale et hospitalière) qui résident et travaillent dans le territoire considéré ;

- la base sociale regroupe les revenus de transferts.

Ces quatre bases (ou types de revenus importés) ne représentent ni les mêmes montants, ni les mêmes enjeux pour les territoires. Les premières estimations des bases économiques réalisées à l'échelle des aires urbaines et des zones d'emplois françaises sur les données de 1999 L. Davezies en 2003, tout comme les améliorations et estimations qui suivront en 2007 
ou en 2016 par M. Talandier conduisent au même résultat, à savoir que la base résidentielle constitue la première source de revenu exogène des territoires.

L'économie résidentielle repose sur des enjeux de population, avant même de parler d'enjeux de consommation. Conséquence de la mobilité des individus et de la déconnexion croissante entre les lieux de production et les lieux de vie, elle est l'une des facettes territoriales du modèle métropolitain et des relations villes-campagnes. L'économie résidentielle est partout. A des degrés et sous des formes variables d'un territoire à l'autre, elle contribue pleinement à leur développement et va dépendre de trois facteurs : la part des actifs pendulaires dans la population, la part et l'attractivité auprès des retraités et l'attrait touristique du lieu. Ainsi, si les espaces périurbains et frontaliers sont spécialisés dans une économie résidentielle de navetteurs, les espaces ruraux le seront plutôt en termes de retraites et pour certains essentiellement de tourisme comme sur les littoraux, dans les espaces de montagne ou dans une large majorité de campagnes situées dans une moitié sud de la France. Les situations sont donc très différentes d'un type de spécialisation à l'autre. En effet, dans certains territoires, notamment ruraux, le poids de l'économie résidentielle peut résulter d'un départ des actifs et du déclin d'activités productives et non d'une réelle attractivité résidentielle et touristique. L'économie résidentielle, au côté des revenus publics et sociaux, devient alors le seul apport de revenus externes. Elle joue dans ce cas un rôle assurantiel à la fois économique et social dans la mesure où elle permet de maintenir un certain niveau d'équipement en commerces et services dans ces espaces.

Inversement, elle peut être le fruit d'une réelle dynamique d'attractivité à même d'assurer le renouveau démographique, social, économique de certains territoires, notamment ruraux (Talandier, 2007). Quoiqu'il en soit, elle n'est ni un mythe, ni un idéal, en rien normative, mais simplement une forme d'économie pour les territoires, un levier d'actions pour les acteurs, avec son lot de conséquences positives et négatives dont il convient de prendre la mesure. Constitutives de l'économie résidentielle, les pensions de retraites apportent une large part des revenus territorialisés.

\section{Répartition et évolution des retraites}

Au 31 décembre 2015, 16 millions de personnes sont titulaires d'une pension de retraite en France. Premier poste de dépenses de la protection sociale, les pensions de vieillesse et de survie s'élèvent à plus de 300 milliards d'euros en 2015, soit un septième du produit intérieur brut (PIB) (Drees, 2017).

Leur poids en volume n'a cessé d'augmenter et occupe une part croissante dans le revenu des ménages. Ainsi, en 2015 , les pensions de retraites représentent près d'un tiers des montants des revenus déclarés aux impôts. Cette part n'était que de $20 \%$ en 2000 Spatialement, la répartition de cette richesse n'est pas homogène. Si l'on regarde les chiffres par grande catégorie d'espace (selon les découpages Insee), on constate le rôle structurant de ces revenus pour les espaces ruraux (table 1 ). 
Table 1 : Répartition spatiale et évolution des retraites entre 2000 et 2015

\begin{tabular}{|c|c|c|c|c|c|}
\hline & Ville-centre & Banlieue & Périurbain & Rural & \begin{tabular}{|r|} 
France \\
métropolitaine \\
\end{tabular} \\
\hline $\begin{array}{l}\text { \% pensions et retraites } \\
\text { dans les revenus déclarés } \\
\text { aux impôts en } 2015\end{array}$ & 31,5 & 28,3 & 28,9 & 37,1 & 30,4 \\
\hline $\begin{array}{l}\text { \% pensions et retraites } \\
\text { dans les revenus déclarés } \\
\text { aux impôts en } 2000\end{array}$ & 22,2 & 18,2 & 15,3 & 23,7 & 20,0 \\
\hline $\begin{array}{l}\text { Evol. Retraites 2000/2015 } \\
\text { (en\%) }\end{array}$ & 41,9 & 55,5 & 88,3 & 56,6 & 52,4 \\
\hline $\begin{array}{l}\text { Evol. nb. pensionnaires } \\
2000 / 2015 \text { (en \%) }\end{array}$ & 19,0 & 30,2 & 38,5 & 18,3 & 24,5 \\
\hline $\begin{array}{l}\text { Montant des pensions / } \\
\text { pensionnaires en } 2015\end{array}$ & 22596 & 24540 & 22576 & 20212 & 22952 \\
\hline $\begin{array}{l}\text { Evol. Montant des pensions } \\
2000 / 2015 \text { (en \%) }\end{array}$ & 19,2 & 19,4 & 36,0 & 32,4 & 22,5 \\
\hline
\end{tabular}

Source : d'après DGI, calculs de l'auteur, 2018

En effet, en 2015 , les retraites représentent $37 \%$ des revenus dans l'espace rural, puis $31,5 \%$ dans les villes-centre, pour descendre à $28,9 \%$ dans le périurbain et $28,3 \%$ dans les banlieues (au sens statistique défini par l'Insee en tant que première couronne des villes-centres). En revanche, c'est dans les espaces périurbains que les volumes de retraites ont le plus progressé en quinze ans, soit une augmentation de près de $90 \%$ ! Dans les espaces ruraux, la hausse a été de $56 \%$. Ces écarts de variation sont dus à une hausse plus rapide du nombre de pensionnaires dans les espaces périurbains, puis dans les banlieues que dans les espaces ruraux où leur nombre progresse finalement moins vite. Le montant des pensions de retraites est assez équilibré d'un type d'espace à l'autre, avec cependant des pensions moins élevées dans le rural, puis dans les villes centre et le périurbain. En revanche, il est intéressant de noter que le montant des pensions par retraité a plus rapidement progressé dans les espaces où il était le plus faible (dans le rural et le périurbain), contribuant ainsi à un rééquilibrage spatial.

Les trois cartes ci-après permettent d'affiner l'analyse territoriale de ces dynamiques.

La première souligne le poids des retraites dans les revenus locaux des espaces ruraux du centre de la France, dans les Pyrénées ainsi que dans les arrière-pays littoraux, soit dans une large partie de la France rurale.

La carte 2 montre les écarts de richesse entre les retraités d'une intercommunalité à l'autre. Les retraites les plus faibles étant dans les EPCI de la région Auvergne, dans certaines collectivités du Nord, au bien encore en Corse ou dans les espaces rétro-littoraux le long de la façade atlantique. Enfin, la carte 3 révèle les évolutions contrastées du montant de ces retraites, avec un certain effet de rattrapage pour les territoires de Bretagne, par exemple, d'une partie des Hauts-de-France, ou bien encore dans le sud du massif-central. 


\section{Conclusion}

Si les retraites sont devenues une source de revenus et de développement économique essentielle pour les territoires, leur impact varie d'un espace à l'autre. Le rural, plus sensible à cette question que $d^{\prime}$ autres espaces n'apparait pas non plus homogène en la matière. Ces disparités révèlent l'enjeu que constitue ces flux de revenus pour l'action locale en matière de développement territorial, enjeu qui pose de nombreuses questions et méritent sans aucun doute une attention toute particulière. 
Carte 1 : Le poids des retraites dans les revenus locaux de 2015 , en $\%$ Echelle des EPCI 2017

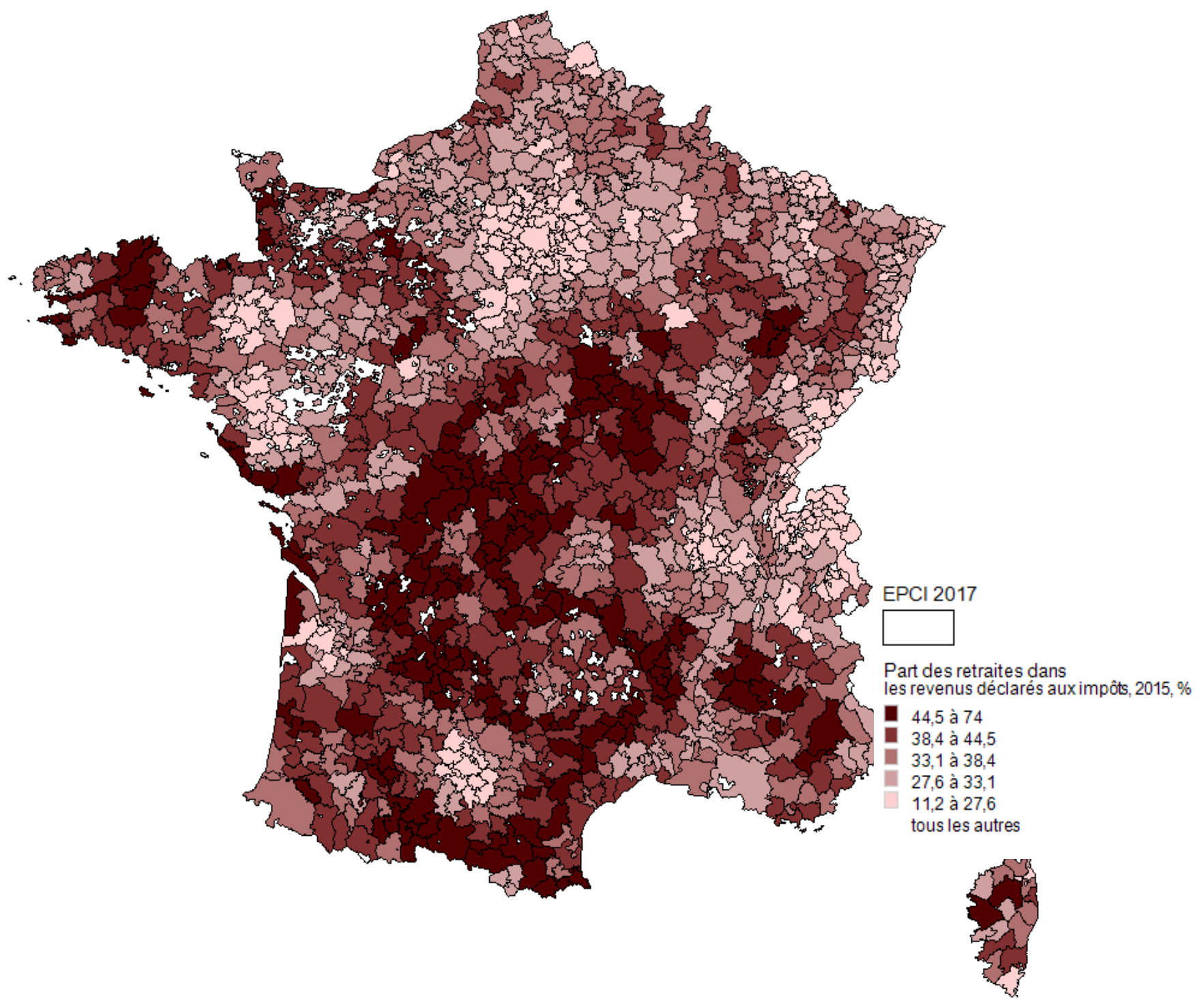

Source : d'après DGI, réalisation de l'auteur, 2018 
Carte 2 : Le montant moyen des retraites par pensionnaire en 2015 , en $€$ Echelle des EPCI 2017



Source : d'après DGI, réalisation de l'auteur, 2018 
Carte 3 : Evolution du montant moyen des retraites entre 2000 et 2015, en \% Echelle des EPCI 2017

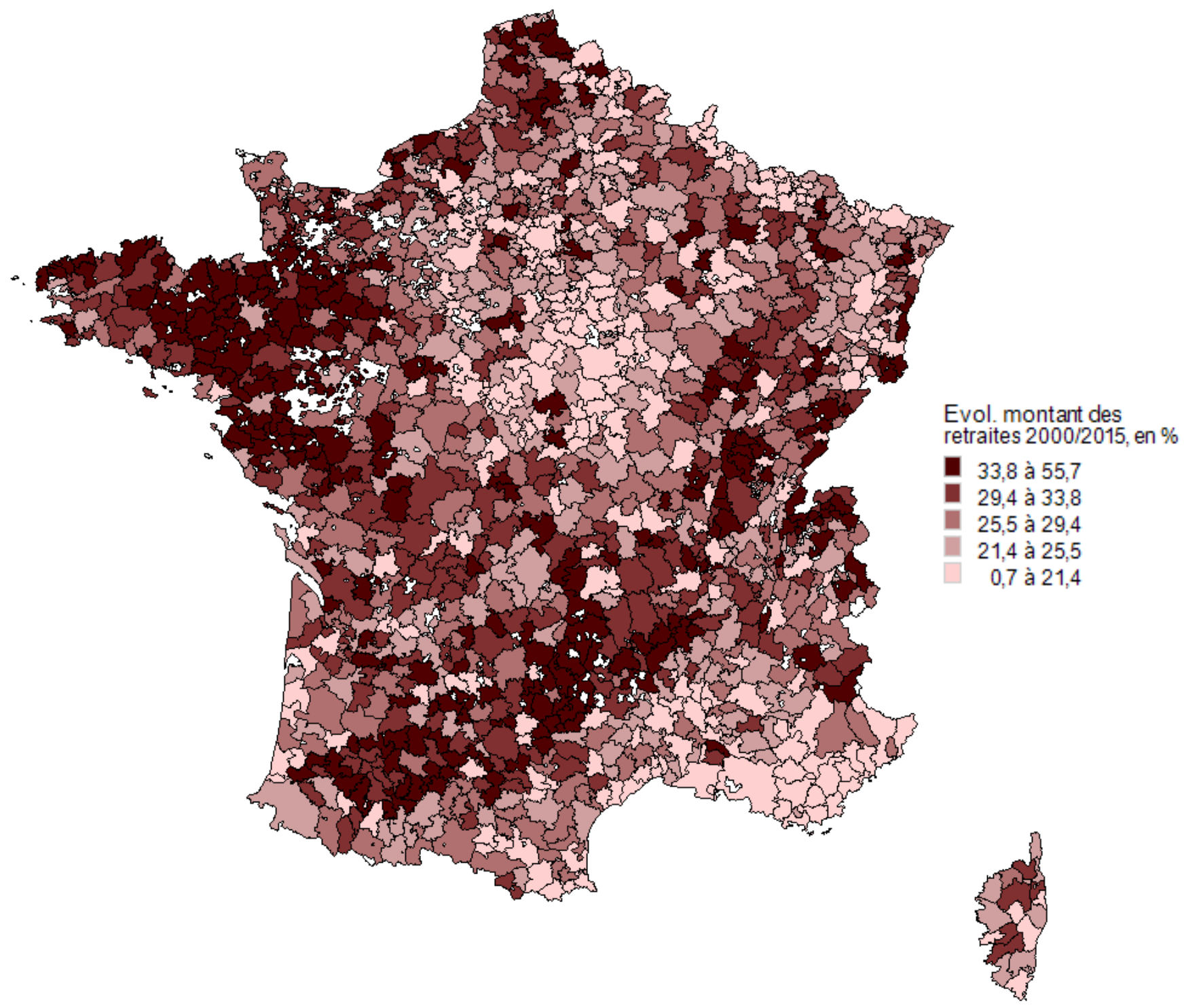

Source : d'après DGI, réalisation de l'auteur, 2018 
Bibliographie

DAVEZIES, L. (2008). LA REPUBLIQUE ET SES TERRITOIRES. LA CIRCULATION INVISIBLE DES RICHESSES. SEUIL.

STOCK, M. (2006). L'HYPOTHESE DE L'HABITER POLY-TOPIQUE: PRATIQUER LES LIEUX GEOGRAPHIQUES DANS LES SOCIETES A INDIVIDUS MOBILES. ESPACESTEMPS. NET.

TALANDIER, M. (2007). UN NOUVEAU MODELE DE DEVELOPPEMENT HORS METROPOLISATION. LE CAS DU MONDE RURAL FRANÇAIS(DOCTORAL DISSERTATION, UNIVERSITE PARIS XII VAL DE MARNE).

TALANDIER, M. (2013). REDEFINIR L'ENJEU DE L'ECONOMIE PRESENTIELLE ET LE ROLE DES FEMMES DANS LES ECONOMIES LOCALES. LE CAS DES TERRITOIRES DE MONTAGNE. JOURNAL OF ALPINE RESEARCH/ REVUE DE GEOGRAPHIE ALPINE, (101-1).

DREES (2017). LES RETRAITES ET LES RETRAITES. PANORAMA DE LA DREES. 